\title{
Absence of Abelian Higgs Hair for Extreme Black Holes
}

\author{
A. Chamblin, ${ }^{1,4}$ J. M. A. Ashbourn-Chamblin, ${ }^{2}$ R. Emparan, ${ }^{3}$ and A. Sornborger ${ }^{4}$ \\ ${ }^{1}$ Institute for Theoretical Physics, University of California, Santa Barbara, California 93106-4030 \\ ${ }^{2}$ Wolfson College, University of Oxford, Oxford OX2 6UD, England \\ ${ }^{3}$ Department of Physics, University of California, Santa Barbara, California 93106 \\ ${ }^{4}$ DAMTP, Silver Street, Cambridge, CB3 9EW, England
}

(Received 13 June 1997)

\begin{abstract}
It has been argued that a black hole horizon can support the long range fields of a Nielsen-Olesen string, and that one can think of such a vortex as black hole "hair." We show that the fields inside the vortex are completely expelled from a charged black hole in the extreme limit (but not in the near extreme limit). This would seem to imply that a vortex cannot be attached to an extreme black hole. Furthermore, we provide evidence that it is energetically unfavorable for a thin vortex to interact with a large extreme black hole. This dispels the notion that a black hole can support "long" Abelian Higgs hair in the extreme limit. [S0031-9007(98)06139-0]
\end{abstract}

PACS numbers: 04.70.-s, 04.40.Nr, 11.27.+d, 98.80.Cq

Black hole "hair" is defined to be any field(s) associated with a stationary black hole configuration which can be detected by asymptotic observers but which cannot be identified with electromagnetic or gravitational degrees of freedom. A number of results have been proven [1] which imply that black holes "have no hair." These results led people to believe that a black hole horizon can only support charges associated with long range gauge fields. However, this prejudice was to some extent discredited when various authors [2], using numerical techniques, discovered black hole solutions of the Einstein-Yang-Mills (EYM) equations that support Yang-Mills fields which can be detected by asymptotic observers (these papers extended the earlier work of Bartnik and McKinnon [3], who found globally regular finite energy solutions in EYM theory without horizons); one therefore says that these black holes are colored. However, these exotic solutions do not impugn the original no-hair results since all such solutions are known to be unstable (see, e.g., Ref. [4]). Since the original no-hair theorems assumed a stationary picture, they simply do not apply to colored holes. On the other hand, colored holes do still exist and so they are said to "evade" the usual no-hair results. These results teach us that we have to tread carefully when we start talking about black hole hair.

With this in mind, we analyze the extent to which hair is present in situations involving topological defects, such as cosmic strings [5]. In [6], evidence was presented that a Nielsen-Olesen (Abelian) vortex can "thread" a Schwarzschild black hole. Inclusion of the gravitational backreaction of a single thin vortex led to a metric which is just a conical defect centered on a black hole [7]. Thus, it was argued that this solution truly is the "thin vortex" limit of a "physical" vortex-black hole configuration. Given these results, one can conclude [6] that the Abelian Higgs vortex is not just dressing for the Schwarzschild black hole, but rather that the vortex is truly hair, i.e., a property of the black hole which can be detected by asymptotic observers.

In this paper, we extend the analysis of [6] and allow the black hole to be charged. That is, we consider an Abelian Higgs vortex in the Reissner-Nordstrom background. In order to "turn up" the charge of the hole, we have to allow for the presence of two U(1) gauge fields (one U(1) is where the charge of the hole lives and the other $\mathrm{U}(1)$ is the symmetry spontaneously broken in the ground state). We find two striking phenomena:

(i) In the extreme limit (but not near extremality) all of the fields associated with the vortex (both the magnetic and scalar degrees of freedom) are expelled from the horizon of the black hole. The magnetic and scalar fields always "wrap around" the horizon in the extremal limit.

(ii) By considering the total energy of the vortex with a black hole inside it, we find an instability as the extreme black hole becomes very large compared to the size of the vortex. Specifically, the energy of a vortex which does not contain the hole inside it is much less than the energy of a vortex which does contain the hole.

In a sense, the behavior (i) was expected, given that extreme black holes generically display such a "Meissner effect," and so can be thought of as "superconductors" (a deeper analysis of the superconducting properties of extremal black holes and $p$-branes will be given in [8]). But from (ii) it follows that a very thin vortex will want to "slide" off of the hole. Thus, the vortex cannot in any way be thought of as a "property of the black hole which can be measured at infinity"; in other words, an Abelian Higgs vortex is not hair for an extreme black hole.

Our treatment of the black hole/string vortex system involves a clear separation between the degrees of freedom of each of these objects. The action takes the form $S=S_{1}+S_{2}$, where $S_{1}$ is an Einstein-Hilbert-Maxwell action for the "background" fields $\left(g_{\mu \nu}, \mathcal{F}_{\mu \nu}\right)$, and $S_{2}$ describes an Abelian Higgs system minimally coupled to 
gravity,

$$
\begin{aligned}
S_{2}= & \int d^{4} x \sqrt{-g} \\
& \times\left(D_{\mu} \Phi^{\dagger} D^{\mu} \Phi-\frac{1}{4 e^{2}} F^{2}-\frac{\lambda}{4}\left(\Phi^{\dagger} \Phi-\eta^{2}\right)^{2}\right) .
\end{aligned}
$$

The degrees of freedom in $S_{2}$ are treated as "test fields." They are the complex Higgs field $\Phi$ and a U(1) gauge field with strength $F_{\mu \nu}$ and potential $A_{\mu}$. The Higgs scalar and the gauge field are coupled through the gauge covariant derivative $D_{\mu}=\nabla_{\mu}+i A_{\mu}$, where $\nabla_{\mu}$ is the spacetime covariant derivative. We choose metric signature $(+---)$. It is also convenient to define the Bogomolnyi parameter $\beta=\lambda / 2 e^{2}=m_{\mathrm{Higgs}}^{2} / m_{\text {vector. }}^{2}$. Notice that we have two different gauge fields: $F$, which couples to the Higgs field and is therefore subject to spontaneous symmetry breaking, and $\mathcal{F}$, which remains massless.

A vortex is present when the phase of $\Phi(x)$ is a nonsingle valued quantity. To better describe this, define the real fields $X, P_{\mu}, \chi$, by $\Phi=\eta X e^{i \chi}$ and $A_{\mu}=P_{\mu}-$ $\nabla_{\mu} \chi$. The vortex is then characterized by $\oint d \chi=2 \pi N$, the integer $N$ being called the winding number.

We will analyze the equations of the vortex in the background of the Reissner-Nordstrom black hole,

$$
\begin{aligned}
d s^{2} & =V d t^{2}-\frac{d \rho^{2}}{V}-\rho^{2}\left(d \theta^{2}+\sin ^{2} \theta d \varphi^{2}\right), \\
V & =1-\frac{2 G m}{\rho}+\frac{q^{2}}{\rho^{2}} .
\end{aligned}
$$

We will work in rescaled coordinates and parameters $(r, E, Q)=\eta \sqrt{\lambda}(\rho, G m, q)$. In these nondimensional variables the Higgs mass is unity. The ReissnerNordstrom black hole has inner and outer horizons where $V(r)=0$. We are interested only in the outer horizon, which is at radius $r_{+}=E+\sqrt{E^{2}-Q^{2}}$. If $r_{+}=E=|Q|$, then $V(r)$ has a double zero at $r_{+}$, and the black hole is said to be extremal.

Return now to the equations of the vortex. One can consistently take $X=X(r, \theta), P_{\varphi}=N P(r, \theta)$, which simplifies the equations of motion to the form

$$
\begin{aligned}
-\frac{1}{r^{2}} \partial_{r}\left(r^{2} V \partial_{r} X\right)- & \frac{1}{r^{2} \sin \theta} \partial_{\theta}\left(\sin \theta \partial_{\theta} X\right)+ \\
& \frac{1}{2} X\left(X^{2}-1\right)+\frac{N^{2} X P^{2}}{r^{2} \sin ^{2} \theta}=0, \\
\partial_{r}\left(V \partial_{r} P\right)+ & \frac{\sin \theta}{r^{2}} \partial_{\theta}\left(\frac{\partial_{\theta} P}{\sin \theta}\right)-\frac{X^{2} P}{\beta}=0 .
\end{aligned}
$$

When $P=1$ (a constant) throughout the space we recover a global string in the presence of the charged hole. Equations (3) and (4) are, in general, intractable in exact form and we need to resort to approximation methods. An analytical solution of these equations for the case where the black hole is small relative to the vortex size is constructed in [9]. There it is seen that both the gauge field and the Higgs field of the vortex have nonzero flux across a nonextreme horizon, but they vanish precisely at the horizon when extremality is reached. Here we resort to numerical integration of Eqs. (3) and (4) outside and on the black hole horizon. (Similar scenarios involving magnetic flux expulsion by extreme black holes have been studied, both numerically and analytically, quite extensively; see, for example, Refs. [10,11,12].)

The Abelian Higgs equations in a background ReissnerNordstrom metric are elliptic. On the horizon they become parabolic. To solve the equations numerically, we use a technique first used by Achúcarro, Gregory, and Kuijken [6]. More details can be found in that reference and in [9]. We have pushed this calculation to the limits, making the vortex as small as we could given the computational constraints. We have found that the vortex is always expelled, no matter how small the magnetic and Higgs flux tubes are taken to be. Here we present a selection of dramatic pictures of the numerical evidence which we have amassed. The behavior shown here holds no matter how small you make the flux tubes. (Note, for the rest of the paper, all of the black holes are assumed to be extreme.)

We begin with the expulsion of the $P$ field by the extreme hole. In Fig. 1 below, we have set $E=Q=10$, with winding number $N=1$ (the smallest winding possible). The Bogomolnyi parameter $\beta$ is set equal to unity, so that the magnetic and Higgs flux tubes are the same size.

Clearly, the $P$ field "wraps" the black hole horizon; furthermore, given the relation between $P$ and $F_{\theta \varphi}$, no magnetic flux is crossing the horizon. The extreme hole

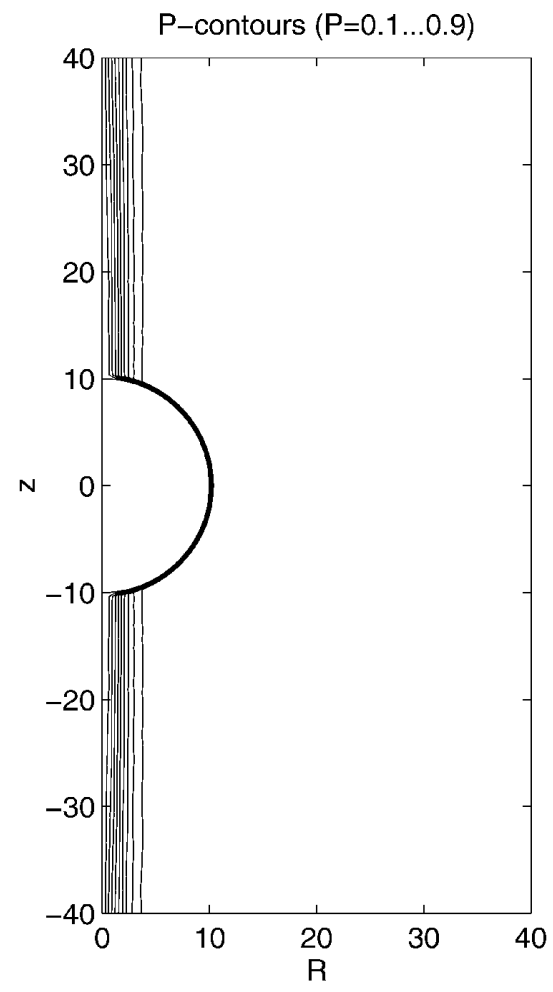

FIG. 1. Expulsion of the $P$ field from the extreme horizon, for the values $E=Q=10, N=1$, and $\beta=1$. 
behaves just like a perfect diamagnet. But can we "puncture" the horizon with flux by making the magnetic flux tube even smaller? The simplest way to make the vector flux tube thinner is by decreasing the value of $\beta$. Since $\beta$ is the ratio of the sizes of the vector and Higgs flux tubes, making $\beta$ very small corresponds to making the magnetic flux tube very skinny. However, we still find the $P$ contours all wrap around the black hole horizon, indicating that there is never any penetration.

We now turn to the behavior of the Higgs field $X$. We have found that the $X$ field is always expelled from the extreme hole, no matter how small the scalar flux tube is made. Actually, in Fig. 2 below, we fix the size of the scalar flux tube (by fixing $N=1$ and $\beta=0.5$ ) and we allow the mass of the extreme hole to increase. The plots run from left to right with increasing mass. The graphs are plotted for the values $E=Q=1,5,10$, and 20 .

The $X$ contours all wrap around the black hole horizon, no matter how large the hole is made. The effect is still true for global strings, where the gauge dynamics is absent. (For figures illustrating flux penetration in the nonextreme limit the reader is referred to [9].)

Now consider the stability of the configurations. Is the black hole stable inside the vortex, or will it try to find its way outside the core? The above sequence provides an intuitive answer to this question. When the black hole is much smaller than the vortex, the black hole is just a "hole," where no vortex energy can be stored. Thus, the hole tends to subtract the total energy of the vortex. On the other hand, when the hole becomes much larger than the vortex, flux stretches to wrap the hole and so we would expect the total energy of the vortex to become very large.

We have computed the total energy $\mathcal{E}_{b h}$ stored in the vortex when it contains a black hole inside it, for different relative sizes of the core and the horizon. (Note, in all numerical calculations we introduce an obvious cutoff, i.e., we do not integrate over all of spacetime to obtain the energy, rather we integrate out to the boundaries of some large "box"- this is justified because the vortex rapidly tends to its flat space form far from the hole.) This is to be compared with the energy of the vortex in the absence of the black hole $\mathcal{E}_{0}$. It is always the case that there exists a maximum mass $E_{\max }$ such that, for all black holes of mass $E<E_{\max }, \mathcal{E}_{b h}(E)<\mathcal{E}_{0}$; as long as the hole is not too massive, it prefers to sit inside the vortex.

The statements above are based on the results of our numerical computations of the total energy $\mathcal{E}_{b h}$. In Fig. 3 below we have plotted the results of one such computation. Here, we have set $\beta=0.5$ and $N=10$. The flat, horizontal line (at 6640) represents $\mathcal{E}_{0}$ in our units (of course, we could always renormalize $\mathcal{E}_{0}$ to 0 since this represents the energy of the background configuration). For these values, $E_{\max }$ is about 15 . Furthermore, for black holes of mass greater than $E_{\max }$ the energy of the vortex is diverging. The erratic behavior of the vortex energy for very small values of the black hole mass is an artifact of the numerical techniques employed in the calculation and should be ignored.

It is clear that a black hole with mass $E>15$ is going to find it energetically favorable to slip out of the vortex. Thus, it is not appropriate to think of such a vortex as a "property of the black hole"; the identification of the vortex as long hair does not go through in this situation. When the mass of the hole is small, one could still try to identify the vortex with hair since, at least in that case, the configuration is energetically stable. On the other hand, the fact remains that the vortex is completely expelled from the hole, even in the (putatively) stable situation. Thus one would say that the vortex is not dressing the black hole. It is not clear to us whether or not one should think of such
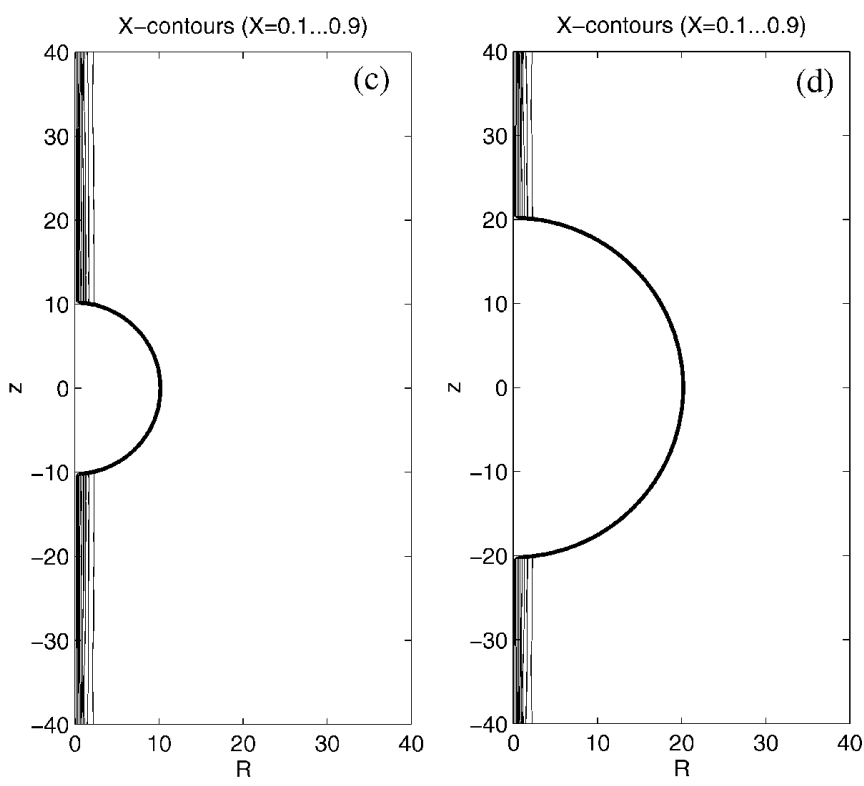

FIG. 2. Expulsion of the Higgs field from the extreme horizon, for the values $E=Q=1$ (a), 5 (b), 10 (c), and 20 (d); $N=1$ and $\beta=0.5$. 


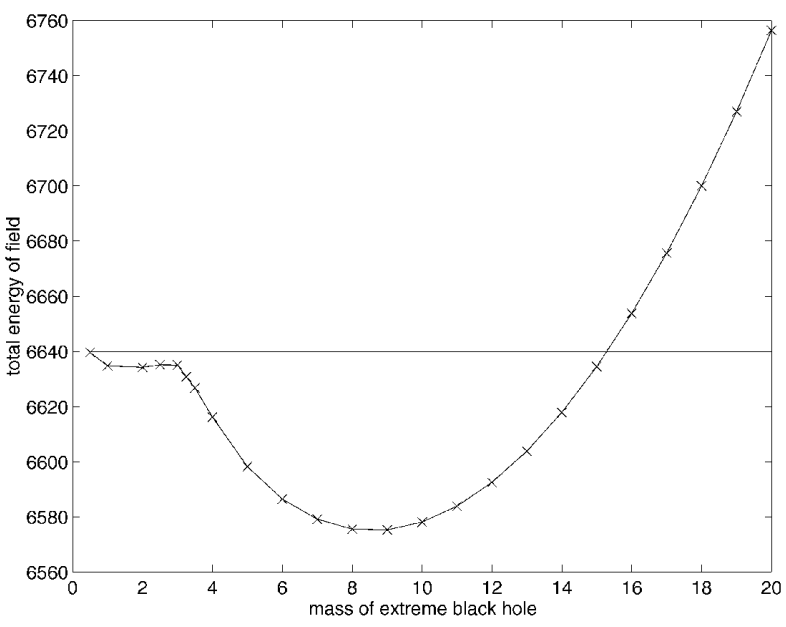

FIG. 3. Plot of total vortex field energy as a function of black hole mass.

a "thick" vortex as genuine hair for a small extreme black hole. This is different from previously studied situations (e.g., the colored black holes), where the black hole may be dressed but the configuration is unstable.

A natural question is whether or not similar results hold when the hole is slightly nonextreme. Our numerical calculations show that, even when the vortex is very thin relative to the radius of the hole, and the charge is very close to extremality, the flux is expelled only in the exact extreme limit (see [9] for more details).

We have provided strong evidence that the fields of a vortex are always expelled from an extreme horizon. Furthermore, a thin enough vortex tends to slip off the black hole. Thus, it appears that an extreme black hole cannot support "long" Abelian Higgs hair. Of course, we have not accounted for the backreaction of the vortex on the geometry. But there is evidence that the expulsion may hold exactly: There exist exact solutions for black holes in $\mathrm{U}(1)^{2}$ theories, where a black hole that is charged to extremality with respect to one of the gauge fields completely expels the field of a (Melvin) flux tube of the other gauge field [8]. This strongly suggests that, after accounting for backreaction, the flux should be expelled from an extreme black hole that sits inside it, at least when the vortex is thick. In any case, backreaction would have to be small if the energy scale of symmetry breaking is small compared to the black hole mass.

We have argued that vortices fail to penetrate extreme horizons. Will this hold true if the string tries to end at a black hole? It has been argued in Ref. [6] that there is no topological obstruction for a topologically stable string to end at a black hole. The argument is still valid for extreme black holes. Imagine then an open string ending on a nonextreme charged horizon (the configuration will be static if we neglect the backreaction of the string). Then increase the charge till the extremal limit, as we have done for the string threading the black hole. There is a crucial difference now: Since a topologically stable string cannot have naked endpoints, when extremality is reached the open string cannot wrap the horizon, or detach from it. It would appear that the string should remain attached to the horizon. This is puzzling, since it is not clear from the local field equations, which determine the expulsion of the field, why the string could end at, but not thread, the black hole. This remains an interesting extension of our work. It could have implications for work in recent years about the pair creation of black holes with strings ending on them [13], and the selection rules on string snapping [14]. A detailed discussion of these results can be found in Ref. [9].

The authors thank Ana Achúcarro, Fay Dowker, Ruth Gregory, and Simon Ross. A. C. was supported by NSF PHY94-07194 at ITP, Santa Barbara, and by Pembroke College, Cambridge. J.M. A. A.-C. was supported by Wolfson College, University of Oxford. R.E. was partially supported by a postdoctoral FPI fellowship (MECSpain) and by Grant No. UPV 063.310-EB225/95. A. S. was supported by U.K. PPARC Grant No. GR/L21488.

[1] W. Israel, Phys. Rev. 164, 1776 (1967); R. B. Wald, Phys. Rev. Lett. 26, 1653 (1971); B. Carter, Phys. Rev. Lett. 26, 331 (1971).

[2] P. Bizon, Phys. Rev. Lett. 64, 2844 (1990); H. P. Künzle and A. K. M. Masood-ul-Alam, J. Math. Phys. 31, 928 (1990); M.S. Volkov and D. V. Gal'tsov, JETP Lett. 50, 345 (1990).

[3] R. Bartnik and J. McKinnon, Phys. Rev. Lett. 61, 141 (1988).

[4] P. Bizon and R. M. Wald, Phys. Lett. B 267, 173 (1991).

[5] A. Vilenkin and E.P.S. Shellard, Cosmic Strings and Other Topological Defects (Cambridge University Press, Cambridge, England, 1994); M. B. Hindmarsh and T. W. B. Kibble, Rep. Prog. Phys. 58, 477 (1995), hep-ph/9411342.

[6] A. Achúcarro, R. Gregory, and K. Kuijken, Phys. Rev. D 52, 5729-5742 (1995), Report No. gr-qc/9505039.

[7] M. Aryal, L. Ford, and A. Vilenkin, Phys. Rev. D 34, 2263 (1986).

[8] A. Chamblin, R. Emparan, and G. W. Gibbons (to be published).

[9] A. Chamblin, J. M. A. Ashbourn-Chamblin, R. Emparan, and A. Sornborger, Can extreme black holes have (long) Abelian Higgs hair?, Report No. gr-qc/9706004.

[10] J. Bicak and L. Dvorak, Phys. Rev. D 22, 2933 (1980).

[11] F. J. Ernst and W. J. Wild, J. Math. Phys. 17, No. 2182 (1976).

[12] Robert M. Wald, Phys. Rev. D 10, 1680 (1974).

[13] S. W. Hawking and S. R. Ross, Phys. Rev. Lett. 75, 3382 (1995), Report No. gr-qc/9506020; R. Emparan, Phys. Rev. Lett. 75, 3386 (1995), Report No. gr-qc/ 9506025; D. M. Eardley, G. T. Horowitz, D. A. Kastor, and J. Traschen, Phys. Rev. Lett. 75, 3390 (1995), Report No. gr-qc/9506041; R. Gregory and M. Hindmarsh, Phys. Rev. D 52, 5598 (1995), Report No. gr-qc/9506054; R. Emparan, Phys. Rev. D 52, 6976 (1995), Report No. gr-qc/9507002.

[14] A. Achúcarro and R. Gregory, hep-th/9705001. 